\title{
Dimensionamento e alocação dinâmica de veículos no transporte rodoviário de cargas completas entre terminais
}

\author{
Rejane Arinos Vasco ${ }^{\mathrm{a}}$, Reinaldo Morabito ${ }^{\mathrm{b} *}$ \\ aPatrus Transportes Urgentes, São Paulo, SP, Brasil \\ bUniversidade Federal de São Carlos, São Carlos, SP, Brasil \\ *morabito@ufscar.br
}

\section{Resumo}

Este artigo trata do Problema da Alocação Dinâmica de Veículos (PADV) no transporte rodoviário de cargas completas entre terminais, incluindo o dimensionamento da frota adicional necessária para o atendimento de demandas em um horizonte de planejamento multi-períodos e finito. O PADV consiste em definir "movimentos" de uma frota de veículos que realiza viagens entre terminais geograficamente dispersos que interagem entre si. Estes movimentos podem ser: veículos carregados com carga completa, vazios para reposicionamento, ou mantidos em um terminal de um período para outro como provisão para o atendimento de demandas futuras. A ênfase é dada na caracterização do problema em situações reais, na modelagem matemática do problema por meio de programação linear inteira e na solução deste utilizando um pacote comercial de otimização. Os experimentos computacionais realizados indicam que essa abordagem pode ser útil para a solução de problemas encontrados no dia a dia de uma empresa transportadora de carga parcelada no Brasil.

Palavras-chave

Alocação dinâmica de veículos. Otimização linear inteira multiperíodo. Transporte rodoviário. Cargas completas de caminhões.

\section{Introdução}

0 presente trabalho tem como objetivo estudar a alocação dinâmica (multiperíodos) de veículos (caminhões) responsáveis pelas transferências de cargas completas entre terminais e propor abordagens de solução baseadas em técnicas de pesquisa operacional, verificando a adequação das soluções obtidas em um caso real de uma típica empresa transportadora rodoviária de cargas no Brasil. É dado enfoque no desenvolvimento de modelos determinísticos que consideram simultaneamente as operações de transporte de transferência de cargas completas entre terminais, uma demanda origem-destino conhecida ou prevista com boa precisão, em um determinado horizonte de planejamento com múltiplos períodos, decisões de alocação de veículos (vazios) visando otimizar o uso dos recursos disponíveis, e no dimensionamento da quantidade de veículos necessários para atender toda a demanda, ao menor custo possível.

0 Problema de Alocação Dinâmica de Veículos (PADV) surge em uma variedade de situações que envolvem o gerenciamento de uma frota de veículos ao longo de um dado período de tempo, possuindo aplicações no transporte de cargas rodoviário, ferroviário, marítimo e aéreo. Para o transporte rodoviário de cargas, um dos primeiros estudos na literatura envolvendo alocação dinâmica de veículos aparece em Powell et al. (1984) no gerenciamento de uma grande frota de caminhões. Um modelo dinâmico não linear, levando em conta as incertezas nas previsões de demanda, foi aplicado. Em seguida, Powell (1986) refinou e estendeu o modelo anterior e incorporou incertezas na quantidade de veículos 
mantidos em estoque, permitindo que veículos ociosos permanecessem em estoque.

Em estudos subsequentes, Powell $(1987,1988)$ apresentaram modelos alternativos para serem usados em ambientes operacionais, na determinação de como gerenciar uma frota de veículos, e também revisaram o PADV no contexto de transporte de cargas completas por caminhões, com atenção especial ao despacho e reposicionamento de caminhões em antecipação a demandas futuras previstas. 0 autor comentou que os modelos determinísticos, apesar de serem mais simples, são viáveis na prática com bom potencial de aplicação. Uma aplicação prática em uma transportadora de carga completa nos Estados Unidos, tratando não somente o PADV, mas também o problema de alocação de motoristas e incorporando incertezas na demanda futura por transportes, foi reportada em Powell (1988). Foi desenvolvida uma ferramenta responsável por combinar em tempo real informações sobre os veículos e cargas com um modelo elaborado de previsão de demanda e de atividade dos veículos, com o objetivo de maximizar a lucratividade e o nível de serviço.

Outros estudos relacionados ao PADV aparecem em Dejax \& Crainic (1987), que estudaram o problema de movimentação de veículos vazios no transporte de cargas, e em Frantzeskakis \& Powell (1990), que desenvolveram heurísticas para solução do PADV com demandas incertas. Outros estudos no modal rodoviário aparecem em Braklow et al. (1992), Powell et al. (1995), Powell (1996), Powell \& Carvalho (1998a, b), Hall (1999) e Hall \& Zhong (2002).

No modal ferroviário, Beaujon \& Turnquist (1991) propuseram um modelo matemático para tratar simultaneamente problemas de alocação dinâmica de veículos com problemas do dimensionamento da frota para o atendimento da demanda. 0 modelo proposto incorporou uma penalização para demandas não atendidas e também incertezas quanto à proporção de veículos disponíveis em cada período e incertezas no tempo de viagem entre terminais. A pesquisa de Beaujon \& Turnquist (1991) foi importante e influente para o desenvolvimento deste trabalho, por ser a primeira iniciativa a tentar modelar simultaneamente as necessidades de frota e a alocação dinâmica de veículos cheios e vazios.

0 modelo de Beaujon \& Turnquist (1991) pode ser visto como um problema de programação estocástica, tendo as demandas e os tempos de viagem como parâmetros aleatórios. De acordo com os autores, essa combinação, além do tamanho do problema a ser resolvido, tornou o modelo intratável para ser solucionado utilizando técnicas tradicionais de otimização estocástica.
Outros estudos relacionados ao PADV no modal ferroviário e outros modais aparecem em Bojovic (2002), Ghiani et al. (2003), Sayarshad \& Ghoseiri (2009) e Sayarshad \& Tavakkoli-Moghaddam (2010).

No presente artigo, o PADV é estudado com foco no transporte rodoviário de cargas completas entre terminais. Convém notar que não foram encontrados trabalhos anteriores na literatura estudando o PADV no contexto do transporte rodoviário de cargas no Brasil. Os movimentos de veículos aqui considerados podem ser: veículos carregados com carga completa, veículos vazios para reposicionamento ou veículos mantidos em um terminal de um período para outro como provisão para o atendimento de demandas futuras.

A ênfase é dada na caracterização do problema em situações reais de transportadoras brasileiras, com base em estudos de caso realizados em Vasco (2012). 0 objetivo é propor modelos matemáticos ajustados aos problemas encontrados em empresas transportadoras rodoviárias de carga no Brasil e analisar as soluções obtidas em situações realistas. Experimentos computacionais são apresentados indicando que a abordagem pode ser útil para apoiar decisões na prática.

Este artigo está organizado da seguinte maneira: na seção 2 são propostos modelos matemáticos de programação linear inteira para representar o PADV, baseados em modelos anteriores da literatura. Porém, os modelos foram modificados e estendidos para incorporar alguns aspectos práticos dos estudos de caso, por exemplo: (i) a situação em que certos grupos de veículos não podem realizar determinadas rotas; (ii) inclusão da decisão de dimensionamento da frota adicional necessária para que toda a demanda seja atendida, (iii) restrições de capacidade de descarga nos terminais, (iv) penalizações por atrasos no atendimento da demanda, entre outros. Na seção 3 são apresentados os resultados obtidos com os experimentos computacionais realizados ao se resolver um conjunto de problemas de tamanho e complexidade similares aos encontrados na prática de uma empresa de transporte rodoviário de cargas parceladas no Brasil. Os resultados apresentados nessa seção foram obtidos utilizando o pacote de otimização CPLEX (1log, 2008). As conclusões e perspectivas para trabalhos futuros são apresentadas na seção 4 .

\section{Modelagem matemática do problema de alocação dinâmica de veículos}

Dentre os modelos revisados na literatura para representar o PADV, utilizou-se o modelo de programação linear apresentado em Ghiani et al. (2003), originalmente formulado em Powell et al. 
(1984) e Powell (1986), como ponto de partida para a modelagem do problema em estudo.

Assim como em Ghiani et al. (2003) e outros estudos da literatura, optou-se pela modelagem determinística para tratar o PADV em função de algumas razões: (i) maior facilidade para compreensão, desenvolvimento e implementação da abordagem de otimização; (ii) os modelos determinísticos são computacionalmente mais fáceis de serem resolvidos do que modelos estocásticos (Birge \& Louveaux, 1997); (iii) esses modelos não necessitam de muitos dados históricos, comonos modelos estocásticos para caracterizar distribuições de probabilidade das variáveis aleatórias envolvidas; e (iv) esses modelos são flexíveis para análises do tipo "what-if", assim como permitem fáceis e importantes análises de sensibilidade e de cenários alternativos. Os modelos determinísticos apresentados a seguir representam razoavelmente bem a prática de certas empresas de transporte e podem ser úteis para apoiar decisões de alocação dinâmica de veículos nestas empresas em nível operacional. Eles foram baseados nas informações e estudos de caso realizados em Vasco (2012).

A seguir são descritos os conjuntos, parâmetros e variáveis utilizados para a modelagem do PADV, com foco na solução do problema prático encontrado em empresas de transporte rodoviário de cargas no Brasil. Os conjuntos usados no modelo são:

Conjunto $\boldsymbol{N}$ de terminais: composto por todos os terminais da rede de transporte rodoviário em estudo, entendendo-se como um terminal ponto onde se originam ou para onde se destinam cargas. Esses terminais podem ainda ter função mista, exercendo também a atividade de consolidação e de transbordo de cargas.

Conjunto $\boldsymbol{E}$ de veículos: formado pela subdivisão de toda a frota disponível em grupos para o transporte de cargas, para distinguir os veículos, seja pela sua forma de remuneração, rotas praticadas, ou ainda como o mais utilizado na prática de algumas transportadoras brasileiras, o tratamento individual de cada veículo, placa a placa. Nesse caso placa a placa, $|E|$ corresponde à quantidade de veículos existentes na frota. A frota foi considerada como "homogênea" apenas no que diz respeito à capacidade transportada de cargas completas entre terminais e desempenho, resultando em velocidades médias equivalentes nos deslocamentos entre terminais.

Conjunto $\boldsymbol{T}$ de períodos: definido a partir do horizonte de planejamento usualmente utilizado em situações práticas, em que são realizados planos semanais, muitas vezes de maneira informal, iniciando na segunda-feira como o primeiro dia para planejamento, e o sábado como o último dia do horizonte de planejamento. Para se aproximar da realidade, tanto dos tempos de viagem entre terminais como também do período para demanda, cada dia pode ser subdividido, por exemplo, em seis períodos de quatro horas (ou menos, dependendo da frequência das decisões envolvidas), totalizando 36 períodos. Uma quantidade menor de períodos, apesar de tornar o problema menor e mais simples de ser resolvido, poderia gerar planos que não poderiam ser utilizados na prática, devido à maior imprecisão nos tempos de viagem entre terminais, e também na janela de tempo requerida para o atendimento da demanda.

\section{Os parâmetros do modelo são:}

- $\tau_{i j}$ : tempo de viagem entre terminais, medido em quantidade de períodos, entre os terminais $i$ e $j$, $i \in N, j \in N$. Em termos práticos, esse parâmetro representa o tempo médio de deslocamento e independe do tipo de veículo utilizado, uma vez que a frota foi considerada "homogênea" em termos de velocidades médias.

- $d_{i j t}$ : demanda por serviços de transporte (em número de veículos cheios) entre os terminais $i$ e $j$ no período $t, i \in N, j \in N, t \in T$. Cada demanda origem-destino consiste em uma carga com peso (volume ou frete) suficiente para que justifique a liberação de um veículo para viagem do terminal de origem para 0 terminal de destino (carga completa).

- $p_{i j}^{v}$ : lucro (receita - custos operacionais diretos) obtido ao realizar a rota de $i$ para $j$, utilizando um veículo do tipo $v, i \in N, j \in N$, e $v \in E$.

- $c_{i j}^{v}$ : custo de deslocamento vazio de veículos do tipo $v$ de $i$ para $j, i \in N, j \in N, v \in E$. Para o caso de frota própria, esse custo está diretamente ligado ao custo operacional, composto por custos fixos e variáveis. 0 custo por quilômetro é calculado pela somatória dos custos fixos dividido pela distância percorrida no período. Já o custo variável é obtido diretamente por quilômetro. Assim, para calcular o valor de $c_{i j}^{v}$ para o caso de frota própria, multiplica-se a distância em quilômetros de $i$ para $j$ pelo custo operacional por quilômetro. Para o caso de veículos terceirizados, há uma negociação prévia do valor a ser pago por quilômetro percorrido, e esse valor é multiplicado pela distância no percurso de $i$ para $j$.

- $C^{v}$ : custo ao adicionar um veículo do tipo $v$ ao sistema, $v \in E$. Esse parâmetro é utilizado nos modelos que realizam o cálculo da quantidade adicional de veículos necessária para o atendimento de toda a demanda (modelos $M_{2}$ e $M_{5}$ a seguir).

- $m_{i t}^{v}$ : quantidade de veículos do tipo (ou grupo) $v$ que entram no sistema no terminal $i$ no período $t, i \in N, t \in T, v \in E$. Observa-se que, em alguns 
modelos matemáticos apresentados a seguir (modelos $M_{2}$ e $M_{5}$ ), esta quantidade de veículos foi definida como sendo uma variável de decisão do modelo, e não um parâmetro de entrada.

- $K_{j t}$ : capacidade de descarga do terminal $j$ (em número de veículos), no período $t, j \in N, t \in T$. Essa capacidade, em termos práticos, pode estar relacionada diretamente com a quantidade de docas disponíveis para descarga de veículos, assim como também da quantidade de equipe interna disponível para tal operação. Essa capacidade varia de terminal para terminal, e ainda por período, pois em um determinado terminal pode haver uma quantidade maior ou menor de docas para descarga e, ainda, diferentes turnos de trabalho, variando ao longo do dia a quantidade de equipes disponíveis para a operação de descarga. Uma equipe de descarga é usualmente composta por um conferente de cargas e dois auxiliares de transportes.

- $h_{i j t}$ : valor unitário de penalização pelo não atendimento da demanda entre terminais $i$ e $j$ no período $t, i \in N, j \in N, t \in T$. Se uma carga não é transportada no período definido, pode incorrer em custos adicionais para o transportador, muitas vezes impostos pelos embarcadores baseados em contratos. Caso haja algum atraso na entrega, o transportador é obrigado a pagar um valor estipulado nesse contrato como "ônus" pelo atraso. Podem ocorrer também custos adicionais nos terminais de destino em decorrência desses atrasos, seja na mão de obra para descarga do veículo de transferência, seja em veículos alocados para entrega, que poderão ficar ociosos.

- $Y_{i j 0}^{v}=0, \forall i \in N, \forall j \in N, \forall v \in E$, inicialização das variáveis definidas a seguir; indica que no período zero não há nenhum movimento de veículo vazio do tipo $v$ entre os terminais $i$ e $j$.

- $A_{i j}^{v}, i \in N, j \in N, v \in E$ sendo:

$A_{i j}^{v}=\left\{\begin{array}{c}1: \text { seo veículo dotipo } v \text { pode realizar o percurso dei para } j \\ 0: \text { casocontrário }\end{array}\right.$

Esse parâmetro é utilizado para representar situações práticas em que certos veículos não realizam determinadas rotas por motivos diversos, por exemplo: (i) rotas que não incluam passagem (na ida ou na volta) ao município de domicílio da família do motorista, (ii) falta de locais específicos para manutenção do veículo, (iii) inexistência de pontos para troca de motoristas, (iv) transportadores autônomos de cargas que nem sempre conhecem todos os percursos, (v) oferta ou escassez de carga de retorno, (vi) condições físicas da rodovia versus condições de manutenção dos veículos, (vii) risco do percurso (ou rota) versus o valor-limite (em unidades monetárias) permitido pela seguradora ao condutor, dentre outros.

As variáveis de decisão do modelo são:

- $X_{i j t}^{v}$ : fluxo (número) de veículos cheios do tipo $v$ usados para satisfazer a demanda $d_{i j t}, i \in N, j \in N$, $t \in T, v \in E$. Vale ressaltar que $X_{i j t}^{v}>0$ somente se $A_{i j}^{v}=1$, caso contrário essa variável não será definida na construção do modelo matemático, ou por simplificação será definida, mas prefixada com valor nulo.

- $Y_{i j t}^{v}$ : fluxo (número) de veículos vazios do tipo $v$ de $i$ para $j$ no período $t, i \in N, j \in N, t \in T, v \in E$. Vale ressaltar que se $i=j$, o movimento é dito de “estoque", e o veículo $v$ é mantido no terminal de origem $i$, de um período a outro. Da mesma forma que em $X_{i j t}^{v}, Y_{i j t}^{v}>0$ somente se $A_{i j}^{v}=1$.

- $I_{i j t}$ : demanda (em número de veículos cheios) não atendida (em backlog) de $i$ para $j$ no período $t$, $i \in N, j \in N, t \in T$. Essa variável é utilizada para computar a quantidade de demanda não atendida em cada período, para cada percurso, e penalizar cargas não atendidas no período da sua solicitação, utilizando o parâmetro $h_{i j t}$ já definido. Caso uma demanda não seja atendida no período em que foi solicitada, ela permanece no terminal de origem aguardando o momento em que será transferida para o terminal de destino. Nesse caso não há "recusa” de carga conforme alguns modelos apresentados, por exemplo, em Powell et al. (1995), pois a realidade de diversas empresas de transporte brasileiras é que, dado que a carga já foi aceita (recebida ou coletada) pelo transportador, ela, em algum momento, deve ser transportada para seu terminal de destino e posterior entrega ao destinatário final.

\subsection{Modelo utilizado para representar o $\operatorname{PADV}\left(M_{1}\right)$}

0 modelo $M_{1}$ a seguir é uma simples modificação do modelo de programação linear em Ghiani et al. (2003). No modelo $M_{1}$ diferente do modelo original apresentado em Ghiani et al. (2003), incorporou-se a possibilidade de utilização de mais de um tipo (ou grupo) de veículos, e também foi feita a inclusão da restrição de que certos tipos (ou grupos) de veículos não podem realizar determinadas rotas. Essas modificações no modelo original tiveram como objetivo representar melhor a realidade prática das empresas transportadoras de carga no Brasil, pois nem todos os veículos podem ser tratados da mesma forma (em um único grupo), por exemplo, (i) veículos próprios, (ii) veículos de terceiros (agregados), (iii) transportador autônomo de carga, entre outros. 
O PADV com múltiplos tipos de veículo (conjunto $E$ ) pode ser modelado como um problema linear de fluxo multicommodity (multifluxo) de mínimo custo, transformando a restrição de atendimento da demanda em uma restrição de mochila (bundle constraint) (Powell et al., 1995). Dessa maneira, diferente do modelo original de programação linear para representar o PADV, ele é de difícil solução exata (NP-difícil) (Ghiani et al., 2003; Vasco, 2012; Vasco \& Morabito, 2014). Considerando os conjuntos, parâmetros e variáveis já definidos, o modelo matemático determinístico para representar o PADV pode ser representado como o seguinte modelo de programação linear inteira, denominado modelo $M_{1}$ :

$$
\max Z_{1}=\sum_{\substack{i \in N \\ \text { i }}} \sum_{\substack{j \neq N \\ i \neq j}} \sum_{v \in E}\left(p_{i j}^{v} X_{i j t}^{v}-c_{i j}^{v} Y_{i j t}^{v}\right)
$$

Sujeito a:

$$
\begin{aligned}
& \sum_{j \in N}\left(X_{i j t}^{v}+Y_{i j t}^{v}\right)-\sum_{\substack{k \in N \\
k \neq i \\
t>\hat{\mathrm{o}}_{i j}}}\left(X_{k i\left(t-\hat{\mathrm{o}}_{i j}\right)}^{v}+Y_{k i\left(t-\hat{\mathrm{o}}_{k i}\right)}^{v}\right)=m_{i t}^{v}+Y_{i i(t-1)}^{v} \\
& \forall i \in N, \forall t \in T, \forall v \in E \\
& \sum_{v \in E} X_{i j t}^{v} \leq d_{i j t}, \forall i, j \in N, \forall t \in T, \\
& X_{i j t}^{v}=0, \text { e } Y_{i j t}^{v}=0, \text { se } A_{i j}^{v}=0, \forall i, j \in N, \forall t \in T, \forall v \in E, \\
& X_{i j t}^{v} \geq 0, Y_{i j t}^{v} \geq 0, \text { inteiros, } \forall i, j \in N, \forall t \in T, \forall v \in E .
\end{aligned}
$$

A função objetivo (1) visa maximizar a diferença entre a receita obtida com o transporte de cargas (movimentos cheios) e o custo ao deslocar veículos vazios para reposicionamento da frota. As restrições (2) impõem a conservação de fluxo no início de cada período, enquanto as restrições (3) asseguram que o número de veículos cheios seja menor ou igual à demanda. Como consequência, temos que as diferenças

$$
\sum_{v \in E} X_{i j t}^{v}-d_{i j t}, \forall i, j \in N, \forall t \in T \text { representam as cargas que }
$$

não puderam ser atendidas. As restrições (3) tornam o modelo $M_{1}$ de multifluxos em cada arco $(i, j)$ em $t$. Caso o problema fosse de monofluxo, envolvendo apenas uma commodity, o problema poderia ser simplesmente modelado e resolvido por programação linear (Ghiani et al., 2003)

0 modelo $M_{1}$ pode ser útil para apoiar decisões de alocação de veículos vazios, selecionando quais demandas deverão ser atendidas, levando-se em conta os diferentes (grupos de) veículos e a contribuição ao lucro da empresa. Por meio da identificação prévia das demandas que não poderão ser atendidas, via modelo, a empresa transportadora pode se antecipar e contratar veículos de terceiros em certos terminais e períodos para que todas as cargas sejam transportadas, sem necessariamente gerar atrasos. A restrição (4) visa garantir que veículos de um dado tipo transitem, cheios ou vazios, em somente um determinado conjunto de percursos. Conforme mencionado anteriormente, essas restrições são relevantes do ponto de vista prático, pois permitem evitar que determinados grupos, ou certas placas de veículos, realizem certas rotas. Por exemplo, direcionar veículos agregados a rotas que possuam frete de retorno. Convém salientar que estas restrições podem ser substituídas por simples procedimentos de fixação de variáveis, disponíveis nos pacotes de otimização, ou ainda no momento da geração do modelo, eliminando essas variáveis do problema.

Convém observar que o modelo $M_{1}$ permite fáceis adaptações e é razoavelmente flexível para incorporar outros aspectos da operação. Por exemplo, nos casos em que parte da demanda $d_{i j t}$ só pode ser atendida por um grupo de veículos $v$ (como, por exemplo, em Green logistics), basta separar essa demanda como um parâmetro específico $d_{i j t}^{v}$ e incluir uma restrição adicional ao modelo, do tipo: $X_{i j t}^{v} \leq d_{i j t}^{v}$. Esse pode ser o caso em que alguns clientes exigem que apenas veículos que atendam às especificações dos seus programas de sustentabilidade sejam utilizados para transportar suas cargas.

\subsubsection{Exemplo ilustrativo}

Para melhor entendimento do modelo $M_{1}$, a seguir utiliza-se um exemplo ilustrativo em Vasco (2012).

Murty é uma transportadora operando na região de Andhraachuki (Índia). No último 11 de julho, quatro requisições de carga completa (TL) foram realizadas: de Chittoor para Khammam em 11 de julho, de Srikakulam para lchapur em 11 de julho, e de Ananthapur para Chittoor em 13 de julho (duas cargas). Em 11 de julho, um veículo estava disponivel em Chittoor e outro em Khammam. Outro veículo estava no momento transportando um carregamento previamente programado e estaria disponível em Chittoor em 12 de julho. Os tempos de viagem entre os terminais são apresentados na Tabela 1. O lucro obtido com um transporte de uma carga completa é 1,8 vezes $o$ custo de transporte de um veículo vazio por período. Sejam $T=\{11$ de julho, 12 de julho, 13 de julho $\}=\{1,2,3\}$ e $N=\{$ Ananthapur, Chittoor, Ichapur, Khammam, Srikakulam $\}=\{1,2,3,4,5\}$, os parâmetros de demanda $(d)$ e de veículos disponíveis $(\mathrm{m})$ podem ser representados como: $d_{531}=1, d_{241}=1$ e $d_{123}=2$, e $m_{21}=m_{41}=m_{22}=1$, respectivamente.

Por simplificação, considere inicialmente o modelo de Ghiani et al. (2003), em que há apenas um tipo de veículo (isto é, $|E|=1$ ) e que não há restrições de circulação em rotas (4)(isto é, $A_{i j}^{v}=1, \forall i, j \in N, v \in E$ ), $\log$, 
o índice $v$ das variáveis e parâmetros do modelo $M_{1}$ pode ser omitido. Uma solução ótima do PADV ((1)-(5)) utilizando-se os dados do exemplo é apresentada na Figura 1, em que as setas associadas as variáveis $X_{i j t} \mathrm{r}$ epresentam movimentos cheios (carregados), e as setas associadas as variáveis $Y_{i j t}$ representam movimentos vazios ou de estoque. Os valores das variáveis na solução ótima são: $X_{241}^{*}=1, X_{123}^{*}=1, Y_{441}^{*}=Y_{442}^{*}=1, Y_{443}^{*}=2 \mathrm{e}$ $Y_{212}^{*}=1$, resultando em um valor da função objetivo de $Z_{1}^{*}=p_{24}+p_{12}-c_{21}=3,6+1,8-1,0=4,4$. Nota-se que as demandas do terminal 5 para o terminal 3 no período $1\left(d_{531}\right)$ e uma das cargas do terminal 1 para o terminal $2\left(d_{123}\right)$ não são atendidas. Ainda nessa figura estão representados os movimentos ditos de "estoque", pois o veículo que entra no terminal 4 no período 1 (parâmetro $m_{41}=1$ ) é mantido neste terminal até o fim do horizonte de planejamento em estudo.

Para ilustrar as implicações e consequências práticas de se incorporar veículos de mais de um tipo, e também a restrição de circulação de veículos de determinados

Tabela 1. Tempos de viagem, custos e lucros entre os terminais do exemplo Murty.

\begin{tabular}{|c|c|c|c|c|c|c|c|c|c|c|}
\hline & \multicolumn{5}{|c|}{$c_{i j}=\tau_{i j}$} & \multicolumn{5}{|c|}{$p_{i j}$} \\
\hline & 1 & 2 & 3 & 4 & 5 & 1 & 2 & 3 & 4 & 5 \\
\hline 1 & 0 & 1 & 2 & 2 & 2 & 0 & 1,8 & 3,6 & 3,6 & 3,6 \\
\hline 2 & 1 & 0 & 2 & 2 & 2 & 1,8 & 0 & 3,6 & 3,6 & 3,6 \\
\hline 3 & 2 & 2 & 0 & 2 & 1 & 3,6 & 3,6 & 0 & 3,6 & 1,8 \\
\hline 4 & 2 & 2 & 2 & 0 & 2 & 3,6 & 3,6 & 3,6 & 0 & 3,6 \\
\hline 5 & 2 & 2 & 1 & 2 & 0 & 3,6 & 3,6 & 1,8 & 3,6 & 0 \\
\hline
\end{tabular}

tipos a certas rotas, o problema exemplo foi alterado da seguinte forma: (i) foi incluído mais um tipo de veículo, portanto, agora temos $|E|=2$; (ii) alterou-se o tipo de veículo que entra no sistema no período 2 no terminal 2, sendo agora um veículo do tipo 2: $m_{22}^{2}=1$; (iii) restringiu-se a circulação de veículos do tipo 2 do terminal 2 para o terminal 1: $A_{21}^{2}=0$.

Com essas alterações, o valor da solução ótima do modelo $M_{1}$ muda para: $Z_{1}^{* *}=p_{24}=3,6$. Como consequência da restrição de circulação $\left(A_{21}^{2}=0\right)$, nenhuma demanda do terminal 1 para o terminal 2 foi atendida ( $d_{123}=2$ ). Na Figura 2, é apresentada de forma gráfica a solução ótima do problema exemplo alterado.

\subsection{Modelo de dimensionamento da frota adicional para operação $\left(M_{2}\right)$}

No modelo $M_{1}$ considera-se que podem ocorrer demandas não atendidas ou rejeitadas. Uma abordagem alternativa para o problema seria: dado um determinado conjunto de cargas a serem transportadas (demandas a serem atendidas), com seus percursos e períodos definidos, e veículos previamente posicionados, o problema pode ser o de dimensionar a quantidade, posição e período de veículos adicionais, necessários para que todas as demandas sejam atendidas. Isso pode ser útil na prática para estimar, por exemplo, as necessidades de contratação de veículos terceirizados (agregados ou terceiros) ao longo do horizonte de

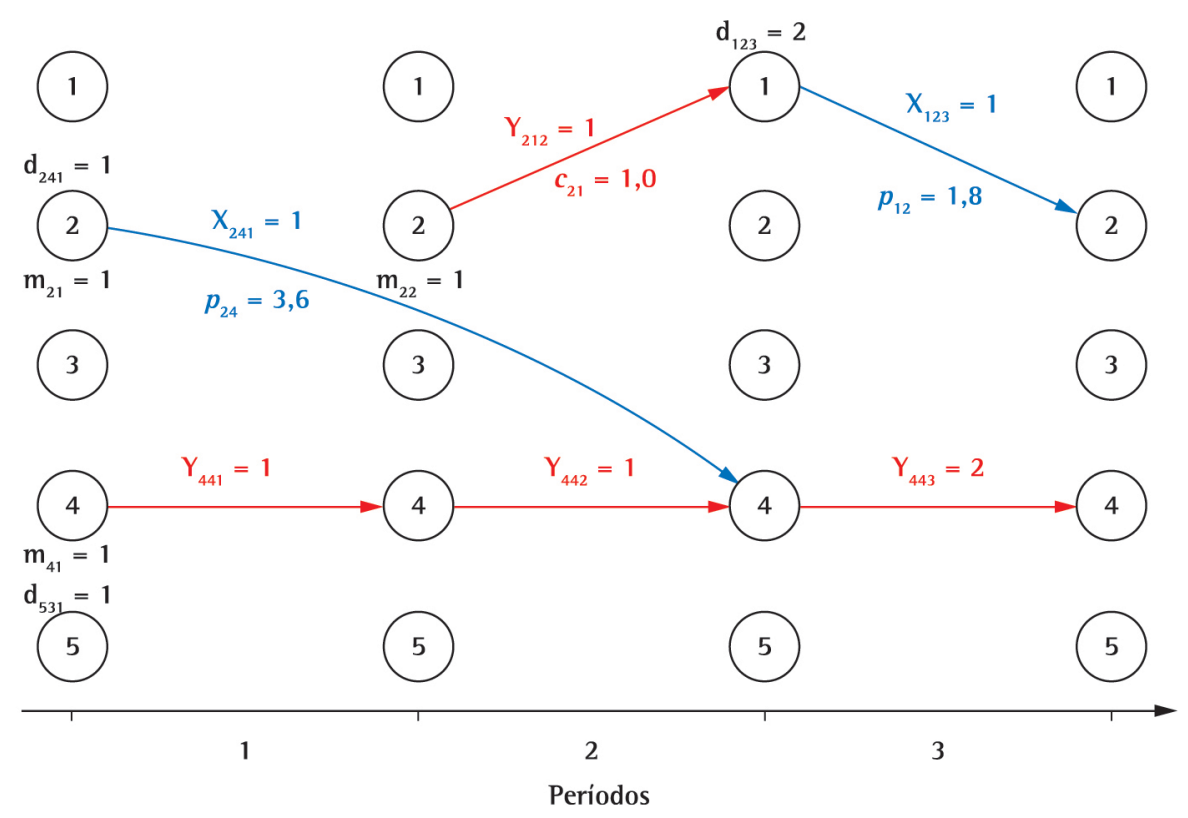

Figura 1. Representação gráfica da solução ótima do problema exemplo. 


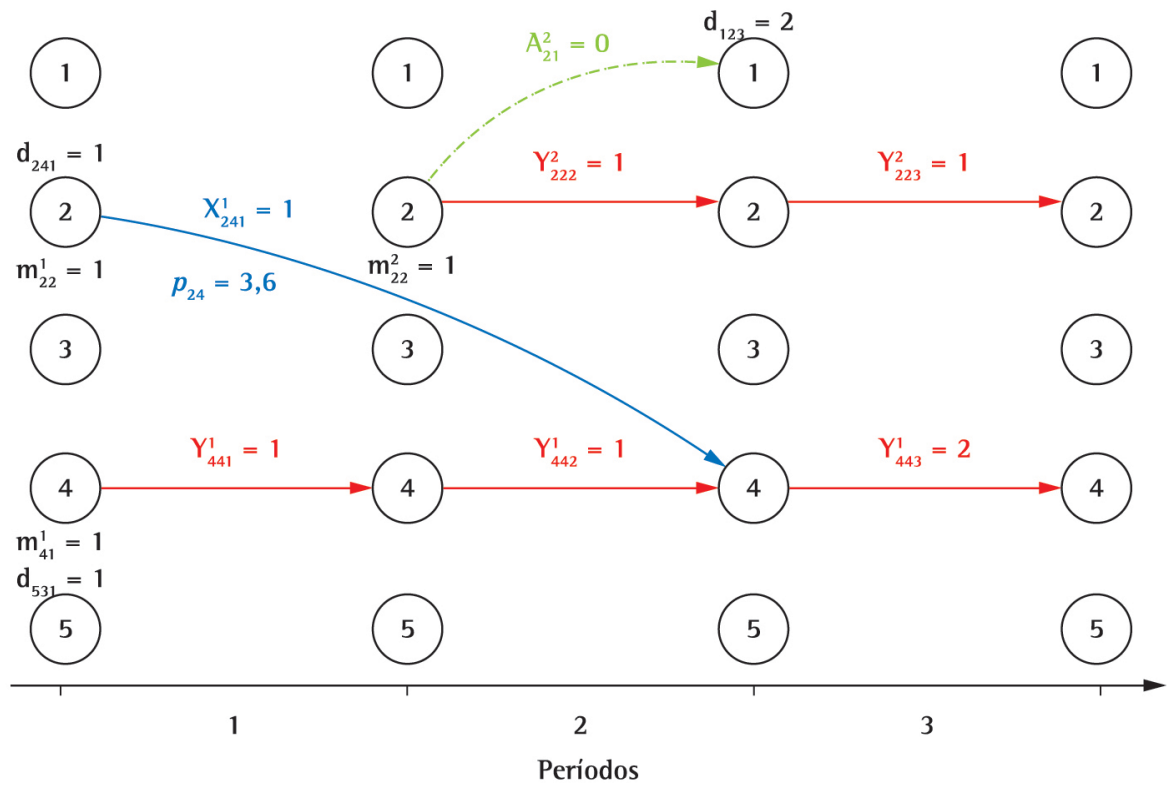

Figura 2. Solução ótima do problema exemplo alterado utilizando-se o modelo $M_{1}$.

planejamento, sendo uma definição ainda no nível de decisão operacional.

Inspirado no trabalho de Beaujon \& Turnquist (1991), a seguir é proposto o modelo determinístico $M_{2}$ para representar o problema de dimensionamento da frota e alocação dinâmica de veículos. Esse modelo permite que questões importantes de planejamento e posicionamento de frota sejam respondidas, como: (i) como e onde os veículos devem ser alocados / distribuídos, (ii) quais os tamanhos desses agrupamentos de veículos e em quais períodos, e (iii) como os veículos devem ser alocados entre movimentos cheios, vazios e agrupamentos. 0 modelo apresentado por Beaujon \& Turnquist (1991) difere do modelo $M_{2}$ em diversos aspectos, por exemplo: (i) os autores apresentam um modelo com parâmetros aleatórios para os tempos de viagem e de demanda, portanto, um modelo estocástico, (ii) a função objetivo incorpora custos fixos e custos ao se manter veículos em estoque de um período a outro em um dado terminal, (iii) penalização de custo por período para uma unidade de demanda não atendida, entre outros.

No modelo $M_{2}, m_{i t}^{v}, i \in N, t \in T, v \in E$, passa a ser uma variável de decisão, em vez de um parâmetro como no modelo $M_{1}$. 0 parâmetro $C^{v}, v \in E$, é considerado para representar o custo ao se alocar um veículo do tipo $v$ ao sistema, e o posicionamento atual (preestabelecido) dos veículos existentes no sistema é representado pelo novo parâmetro $\bar{m}_{i t}^{v}, i \in N$, $t \in T, v \in E$. Assumindo os parâmetros e variáveis já definidos, o modelo $M_{2}$ pode ser escrito como o seguinte problema de programação linear inteira:

$$
\min Z_{2}=\sum_{i \in N \in t \in T} \sum_{v \in E}\left(C^{v}\left(m_{i t}^{v}-\bar{m}_{i t}^{v}\right)\right)+\sum_{i \in N} \sum_{j \in N} \sum_{t \in T} \sum_{v \in E} c_{i j}^{v} Y_{i j t}^{v}
$$

Sujeito a:

(2), (4), (5),

$\sum_{v \in E} X_{i j t}^{v}=d_{i j t}, \forall i, j \in N, \forall t \in T$

$m_{i t}^{v} \geq \bar{m}_{i t}^{v}, \forall i \in N, \forall t \in T, \forall v \in E$

$m_{i t}^{v}>0$, inteiros, $\forall i \in N, \forall t \in T, \forall v \in E$

A função objetivo (6) visa minimizar o custo de se alocar veículos adicionais para a operação, adicionado aos custos associados ao deslocamento de veículos vazios. A restrição (2) é a equação de balanço de veículos para um dado terminal, em determinado período e para cada tipo de veículo. A restrição (4), da mesma forma que no modelo $M_{1}$, visa garantir que veículos transitem somente em rotas permitidas, independentemente se o transporte é vazio ou carregado. Já a restrição (7) assegura que toda a demanda seja atendida, assumindo que a demanda não é restrita a um tipo específico de veículo. A restrição (8) assegura que os veículos já existentes no sistema sejam considerados para o dimensionamento das necessidades de frota.

Considere novamente o problema exemplo da seção 2.1 , com as seguintes alterações: 
- $C^{1}=10,0$ e $C^{2}=11,5$;

- inclusão de mais um tipo de veículo, sendo agora $|E|=2$;

- alteração do tipo de veículo que entra no sistema no período 2 no terminal 2, sendo agora um veículo do tipo 2: $m_{22}^{2}=1$;

- restrição da circulação de veículos do tipo 2 do terminal 2 para o terminal 1: $A_{21}^{2}=0$.

Uma solução ótima desse problema exemplo alterado, definindo simultaneamente a alocação e o dimensionamento veículos e utilizando o modelo $M_{2}$, é apresentada na Figura 3. É importante destacar que, ao levar em consideração o parâmetro relativo à frota existente no problema $\left(\bar{m}_{21}^{1}=\bar{m}_{41}^{1}=\bar{m}_{22}^{2}=1\right)$, de acordo com a restrição (8), as seguintes variáveis foram limitadas inferiormente:

$m_{21}^{1} \geq 1$,

$m_{41}^{1} \geq 1$,

$m_{22}^{2} \geq 1$,

0 valor da solução ótima obtida é:

$Z_{2}^{*}=C^{1} \times\left(\left(m_{51}^{1}-\bar{m}_{51}^{1}\right)+\left(m_{13}^{1}-\bar{m}_{13}^{1}\right)+\left(m_{41}^{1}-\bar{m}_{41}^{1}\right)+\left(m_{21}^{1}-\bar{m}_{21}^{1}\right)\right)$

$+C^{2} \times\left(m_{22}^{2}-\bar{m}_{22}^{2}\right)+c_{41}^{1} \times Y_{411}^{1}$

$=10,0 \times((1-0)+(1-0)+(1-1)+(1-1))+11,5 \times(1-1)+(2 \times 1)$

$=10,0 \times 2+2$

$=22$.

\subsection{Modelo incorporando restrições de capacidade nos terminais $\left(M_{3}\right)$}

A capacidade de operação de terminais de carga parcelada que operam como um cross-docking (Oliveira \& Pizzolato, 2002) está diretamente relacionada à quantidade máxima possível de veículos de transferência que podem ser descarregados em um dado período de tempo. Fatores relacionados à estrutura operacional, como a quantidade de docas para descarga, mão de obra envolvida e tecnologia empregada, são fundamentais para a definição da capacidade de um terminal.

Visando adequar o modelo matemático $M_{1}$ à realidade de empresas transportadoras de carga parcelada, foi incorporada ao modelo a restrição de capacidade de cada terminal em um dado período, representado pelo parâmetro $K_{j t}$, sendo relacionada explicitamente à quantidade máxima de veículos de transferência que podem ser descarregados simultaneamente no terminal $j, j \in N$, em um dado período $t, t \in T$. Sejam os mesmos conjuntos, parâmetros e variáveis já definidos. 0 modelo $M_{3}$ pode ser escrito como o modelo $M_{1}$, incorporando somente a seguinte restrição:

$$
\sum_{\substack{i \in N \\ i \neq j \\ t>\hat{\mathrm{o}}_{i j}}} \sum_{v \in E} X_{i j\left(t-\hat{\mathrm{o}}_{i j}\right)}^{v} \leq K_{j t}, \forall j \in N, \forall t \in T
$$

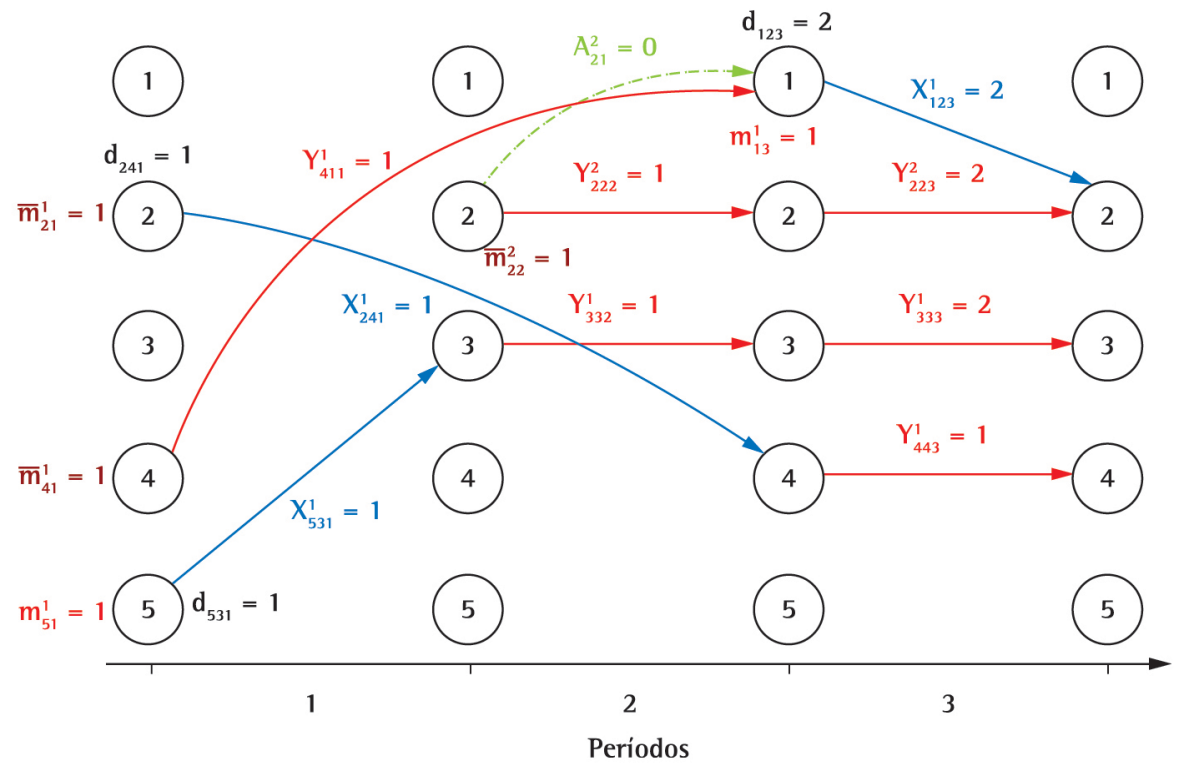

Figura 3. Solução ótima do problema exemplo alterado utilizando-se o modelo $M_{2}$. 


\subsection{Modelo de reposicionamento de veículos com backlog da demanda $\left(M_{4}\right)$}

0 modelo matemático $M_{1}$ utilizado para representar o PADV, representado pelas Equações 1-5, permite que cargas sejam rejeitadas. Em situações práticas do transporte de cargas parceladas, é comum que demandas não atendidas em um dado período, por falta de veículo, aguardem no terminal de origem para serem transportadas em um período posterior (em backlog). Ou seja, são transportadas para o terminal de destino, mesmo que com atrasos, pois em algum momento deverão ser atendidas.

Sendo assim, é proposto o modelo matemático $M_{4}$, a partir do modelo $M_{1}$, que incorpora a situação de cargas não atendidas no período em que ocorreu a demanda, penalizando os atrasos decorrentes da falta de veículos para transporte. Neste modelo $M_{4}$ é incluído o parâmetro $h_{i j t}$, correspondendo ao valor unitário de penalização pelo não atendimento da demanda entre terminais $i$ e $j$ no período $t, i \in N, j \in N$, $t \in T$. E ainda é incluída a variável $I_{i j t}$, a demanda não atendida de $i$ para $j$ no período $t, i \in N$, $j \in N, t \in T$.

Sejam os mesmos conjuntos, parâmetros e variáveis já definidos. 0 modelo $M_{4}$ utilizado para representar o PADV, permitindo backlog de cargas, pode ser descrito como o seguinte problema de programação linear inteira:

$$
\max Z_{4}=\sum_{\substack{i \in N \\ i \neq j}} \sum_{\substack{j \in N \\ i \neq T}} \sum_{v \in E}\left(p_{i j}^{v} X_{i j t}^{v}-c_{i j}^{v} Y_{i j t}^{v}\right)-\sum_{\substack{i \in N \\ j \neq N}} \sum_{\substack{j \in N \\ j \neq i}} h_{i j t} I_{i j t}
$$

Sujeito a:

$$
\text { (2), (4), (5), (10), }
$$

$$
\begin{aligned}
& I_{i j t}=I_{i j(t-1)}+d_{i j t}-\sum_{v \in E} X_{i j t}^{v}, \forall i, j \in N, \forall t \in t|t\rangle 1, \\
& I_{i j T}=0, \forall i, j \in N \\
& I_{i j 1}=d_{i j 1}-\sum_{v \in E} X_{i j 1}^{v}, \forall i, j \in N \\
& I_{i j t} \geq 0, \text { inteiros }, \forall i, j \in N, \forall i \in T
\end{aligned}
$$

A função objetivo (11) visa maximizar a receita obtida ao se realizar o transporte de cargas, deduzindo-se os custos para reposicionamento de veículos vazios e a penalização pelo não atendimento de demandas no período requerido. A restrição (12) representa a quantidade de demanda não atendida para um dado percurso e período. A restrição (13) garante que toda a carga deve ser transportada até o último período. A restrição (14) garante que não haja backlog no primeiro período.

Considere novamente o problema exemplo da seção 2.1 , já com as modificações de parâmetros incorporadas conforme apresentado para o modelo $M_{3}$, em que foram fixados os parâmetros $m_{22}=2$, e $K_{24}=1$. Foi incorporada ainda no problema exemplo a penalização por demandas não atendidas, cujo valor foi fixado em 0,5 para qualquer demanda, ou melhor, $h_{i j t}=0,5, \forall i, j \in N, \forall t \in T .0$ valor da solução ótima do modelo $M_{4}$ é: $Z_{4}^{*}=5,3$, e a representação gráfica, bem como o valor das variáveis de decisão maiores que zero, são apresentadas na Figura 4. Para facilitar a visualização, a figura foi estendida até o período 5 e, como se utilizou um único grupo de veículos $(|E|=1)$, por simplicidade o índice $v$ foi omitido das variáveis e parâmetros.

$Z_{4}^{*}=p_{24} X_{241}+p_{12} X_{123}+p_{12} X_{124}+p_{53} X_{533}$

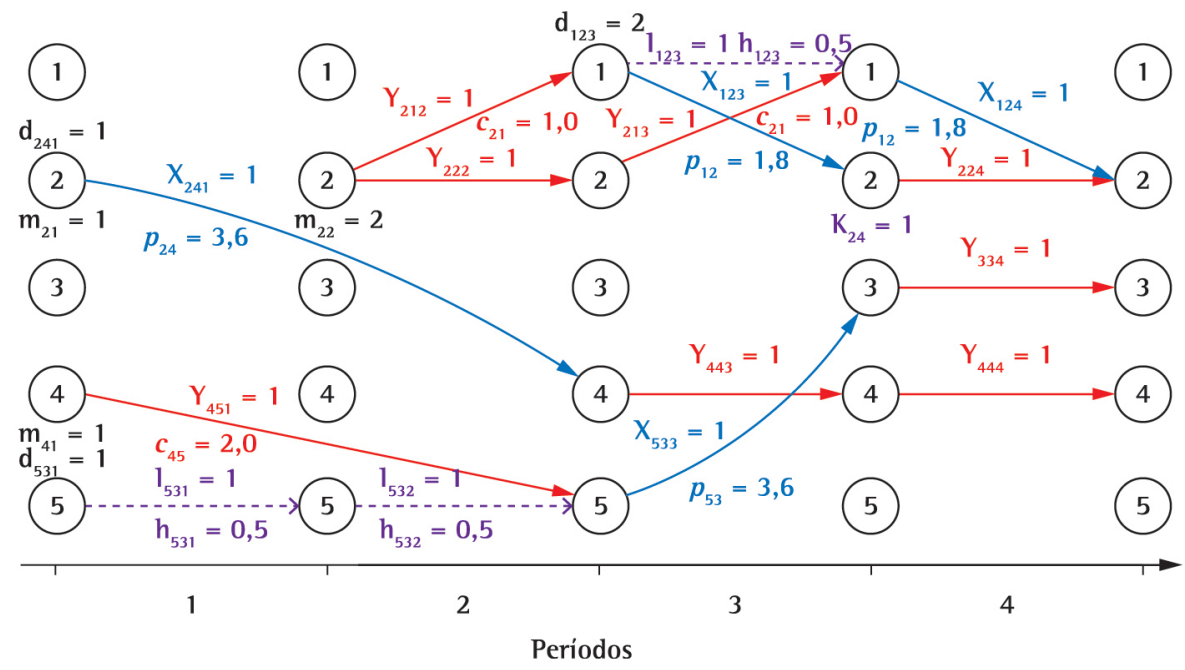

Figura 4. Solução ótima do problema exemplo para o modelo $M_{4}$. 
$-\left(c_{45} Y_{451}+c_{21} Y_{212}+c_{21} Y_{213}\right)$

$-\left(h_{531} I_{531}+h_{532} I_{532}+h_{123} Y_{123}\right)$

$=(3,6 \times 1+1,8 \times 1+1,8 \times 1+3,6 \times 1)$

$-(2 \times 1+1 \times 1+1 \times 1)$

$-(0,5 \times 1+0,5 \times 1+0,5 \times 1)$

$=10,8-4,0-1,5=5,3$.

Pela figura é possivel verificar que toda a demanda foi atendida, porém a demanda $d_{531}=1$ não é atendida por não existir veículo disponível, e a demanda $d_{123}=1$, devido a sua capacidade $K_{24}=1$, também não pôde ser atendida no período demandado, sendo atendida em um ou dois períodos posteriores, havendo portanto o backlog da demanda, sendo evidenciado ainda pelo valor das variáveis: $I_{531}^{*}=1 ; I_{532}^{*}=1 ; I_{123}^{*}=1$.

\subsection{Modelo de dimensionamento da frota com backlog da demanda $\left(M_{5}\right)$}

0 modelo $M_{5}$, assim como o modelo $M_{2}$, avalia as necessidades de frota adicional necessária para operação (ou seja, $m_{i t}^{v}$ é variável de decisão do modelo), e permite backlog de cargas, podendo ser representado a partir dos modelos $M_{2}$ e $M_{4}$. Considerando-se os mesmos conjuntos, parâmetros e variáveis definidos, o modelo $M_{5}$ é definido por:

$\min Z_{5}=\sum_{i \in N} \sum_{t \in T} \sum_{v \in E}\left(C^{v}\left(m_{i t}^{v}-\bar{m}_{i t}^{v}\right)+\sum_{\substack{j \in N \\ i \neq j}}\left(c_{i j}^{v} Y_{i j t}^{v}+h_{i j t} I_{i j t}\right)\right)$

sujeito a:

(2), (4)-(5), (8), (9), (10), (12)-(15).

A função objetivo (16) visa minimizar os custos envolvidos ao se atribuir veículos adicionais para operação, somados à penalização por demandas não atendidas no período solicitado. Incorporadas ao modelo $M_{5}$, temos as restrições de balanço da quantidade de veículos em cada terminal e em cada período (2), e também as restrições de circulação de certos veículos em determinadas rotas, representadas pela Equação 4. A integralidade das variáveis de decisão do modelo é garantida pelas restrições (5), (9) e (15). A restrição (12) define a quantidade de demanda não atendida entre terminais em um dado período. As restrições que asseguram que não haja backlog no primeiro e último períodos são representadas pelas Equações 13 e 14, respectivamente. A restrição (10) diz respeito à capacidade de cada terminal em cada período, que não deve ultrapassar um dado limite.

Voltando ao problema exemplo da seção 2.1, e considerando os mesmos parâmetros já incluídos anteriormente e utilizados pelos modelos $M_{2}, M_{3}$ e $M_{4}$, obtemos a seguinte solução ótima para o modelo $M_{5}$, representada de forma gráfica na Figura 5. 0 valor dessa solução é:

$$
\begin{aligned}
& Z_{5}^{*}=C^{1} \times\left(m_{21}^{1}-\bar{m}_{21}^{1}\right)+C^{1} \times\left(m_{41}^{1}-\bar{m}_{41}^{1}\right)+C^{2} \times\left(m_{22}^{2}-\bar{m}_{22}^{2}\right) \\
& +\left(c_{21}^{2} Y_{212}^{2}+c_{21}^{1} Y_{213}^{1}+c_{45}^{1} Y_{451}^{1}\right) \\
& +\left(h_{123} I_{123}+h_{241} I_{241}+h_{242} I_{242}+h_{243} I_{243}+h_{531} I_{531}+h_{532} I_{532}\right) \\
& =10,0 \times(1-1)+10,0 \times(1-1)+11,5 \times(1-1) \\
& +(1 \times 1)+(1 \times 1)+(2 \times 1) \\
& +(0,5 \times 1+0,5 \times 1+0,5 \times 1+0,5 \times 1+0,5 \times 1+0,5 \times 1) \\
& =0,0+4,0+3,0=7,0 .
\end{aligned}
$$

Na Figura 5, é possivel verificar que toda a demanda foi atendida sem a necessidade de veículos adicionais, porém existindo backlog de demandas não atendidas, principalmente a demanda no primeiro período do

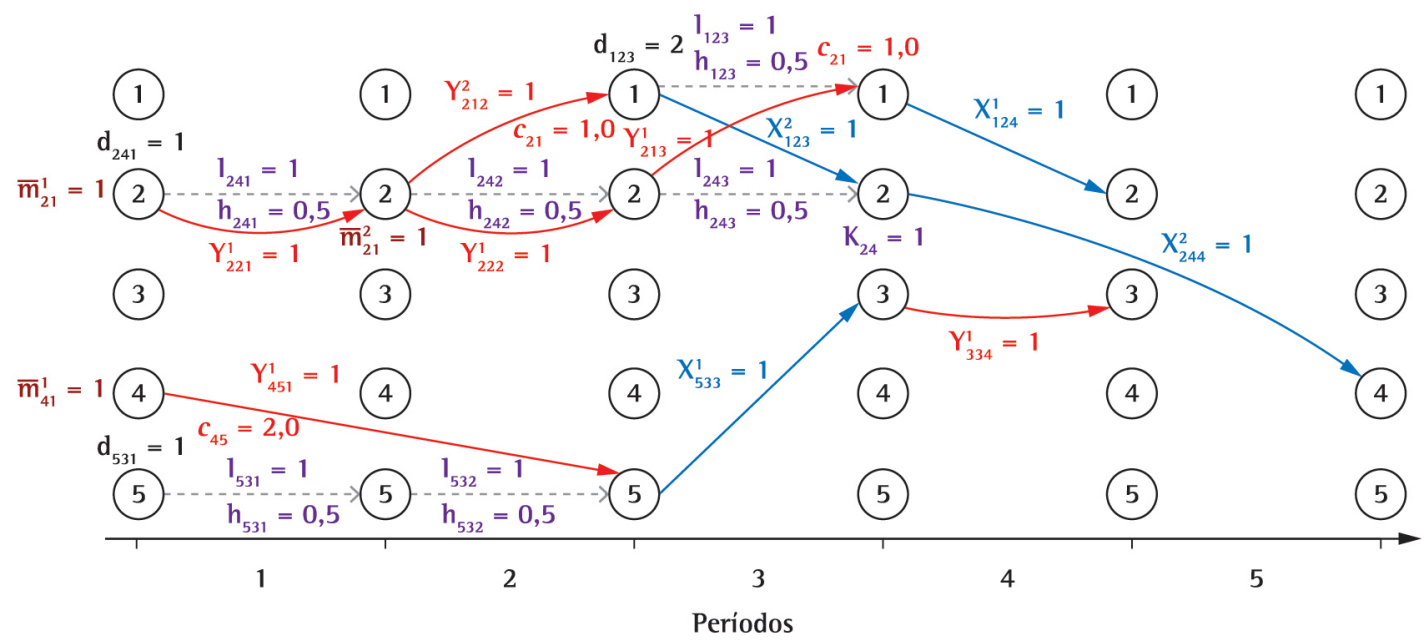

Figura 5. Solução ótima do problema exemplo para o modelo $M_{5}$. 
terminal 2 para o terminal $4, d_{241}=1$, que foi atendida somente no período 4. Outro backlog foi de uma das duas cargas do terminal 1 para o terminal 2 no período $3, d_{123}=2$, que foi atendida no período 4 . É interessante ressaltar que, em situações práticas, nem sempre é possivel, principalmente para certos percursos considerados de curta distância, fazer com que cargas aguardem mais que 2 períodos para serem transportadas. 0 correto balanceamento do parâmetro de penalização por demandas não atendidas ( $h_{i j t}, i \in N, j \in N, t \in T$ ) é um fator crucial, pois interfere diretamente no dimensionamento da frota para operação, que possui um custo proporcionalmente elevado.

\section{Experimentos e resultados computacionais}

Nesta seção são apresentados os experimentos computacionais realizados visando analisar o desempenho e a aplicabilidade dos modelos $M_{1}-M_{5}$ em problemas de tamanho e complexidade similares aos encontrados na prática de uma empresa transportadora de carga. Inicialmente foram realizados experimentos com dados reais de uma empresa transportadora de carga parcelada que opera pelo modal rodoviário no Brasil. Com o objetivo de avaliação dos modelos propostos, considerou-se inicialmente um problema de menor porte, utilizado como exemplo-teste, possuindo 36 períodos ( 6 períodos de 4 horas cada, em 6 dias); 02 grupos de veículos: agregados e próprios $(|E|=2$ ). Os outros parâmetros dos modelos, por exemplo, a capacidade dos terminais $\left(K_{j t}\right)$, penalização por backlog $\left(h_{i j t}\right)$, circulação $\left(A_{i j}^{v}\right)$, foram variados convenientemente para avaliar a sua influência no resultado final obtido.

Um exemplo dos resultados obtidos ao resolver o problema-teste utilizando o modelo $M_{5}$ é apresentado na Tabela 2, em que se pode visualizar a influência do parâmetro $h_{i j t}$ na solução do problema. Quanto maior a penalização, menor a quantidade de movimentos de backlog, maior a quantidade de veículos necessária para que toda a demanda seja atendida, e também, uma maior quantidade de movimentos de "estoque". Mais detalhes sobre esses e outros experimentos são apresentados em Vasco (2012).

Após os experimentos iniciais com um problema de pequeno porte, desenvolveu-se um programa usando a linguagem de programação $C$ para gerar aleatoriamente os problemas, que em seguida foram resolvidos utilizando-se os modelos propostos na seção $2\left(M_{1}, M_{2}, M_{3}, M_{4}\right.$ e $\left.M_{5}\right)$. Foram gerados cinco problemas aleatórios para cada modelo, totalizando 25 problemas a serem resolvidos, com os seguintes parâmetros para o programa gerador de problemas aleatórios:

- 36 períodos (6 períodos de 4 horas cada, em 6 dias);

- 53 terminais;

- 300 percursos com cargas a serem transportadas, sendo em cada percurso até 10 cargas;

- 2 grupos de veículos: (i) próprios, e (ii) agregados $(|E|=2)$;

- 130 ofertas de veículos distribuídos nestes dois grupos, com $m_{i t}^{v}$ variando para cada $v$ uma quantidade de até 10 veículos para um dado $i$ e $t$, sendo $\sum_{i \in N} \sum_{t \in T} \sum_{v \in E} m_{i t}^{v}=130$;

sendo esses valores inspirados em problemas reais com dimensões similares às encontradas na prática (para mais informações desses problemas, ver Vasco, 2012). Para os parâmetros:

- Capacidade dos terminais $\left(K_{j t}\right)$ : foram gerados valores aleatórios inteiros positivos entre 9 e 18, para cada terminal $j$ em cada período $t, \forall j \in N$, $\forall t \in T$.

- Penalização por backlog $\left(h_{i j t}\right)$ : foram gerados valores reais positivos aleatórios entre 0,1 e $1,1, \forall i, j \in N$, e $\forall t \in T$.

- Custo por tipo de veículo $\left(C^{v}\right)$, sendo um valor real positivo aleatório entre 0,5 e $10,5, \forall v \in E$.

- Custo de reposicionamento de veículos vazios $\left(c_{i j}^{v}\right)$ : valor real positivo aleatório entre 1 e 9 , sendo gerado individualmente para cada combinação de $i, j$ e $v, i, j \in N, v \in E$.

- Lucro ao se transportar veículos cheios $\left(p_{i j}^{v}\right)$, um valor real positivo aleatório entre 10 e 18 , gerado para cada $i, j \in N$, e para cada $v \in E$.

- 0 tempo de viagem entre terminais $\left(\hat{o}_{i j}\right)$ foi calculado da seguinte forma:

- são gerados aleatoriamente $|N|$ pontos no plano, com abscissas e ordenadas entre 1 e 100, assumindo somente valores inteiros;

- é criada uma matriz de distâncias euclidianas entre os pontos gerados, sendo a cada distância $d$ calculada, $\hat{o}_{i j}=\frac{d}{15}$.

- 0 parâmetro $A_{i j}^{v}$ foi variado convenientemente, sorteando aleatoriamente a possibilidade de o veículo $v$ poder realizar o percurso de $i$ para $j\left(A_{i j}^{v}=1\right)$, $\forall i, j \in N, \forall v \in E$.

Os limites utilizados para a geração aleatória dos valores dos parâmetros foram similares aos encontrados 
Tabela 2. Resultados obtidos variando-se o parâmetro $\boldsymbol{h}_{\boldsymbol{i j t}}$

\begin{tabular}{lccc} 
para o modelo & $\boldsymbol{M}_{5}$ & & \\
\hline \multicolumn{1}{c}{ Item } & $h_{i j t}=50,00$ & $h_{i j t}=200,00$ & $h_{i j t}=400,00$ \\
\hline $\begin{array}{l}\text { Função } \\
\text { Objetivo }\end{array}$ & $85.243,00$ & $91.112,00$ & $95.014,00$ \\
$\begin{array}{l}\text { Demanda } \\
\text { atendida }\end{array}$ & 114 & 114 & 114 \\
$\begin{array}{l}\text { Demanda não } \\
\text { atendida }\end{array}$ & 0 & 0 & 0 \\
$\begin{array}{l}\text { Movimentos } \\
\text { cheios }\end{array}$ & 114 & 114 & 114 \\
$\begin{array}{l}\text { Movimentos } \\
\text { vazios }\end{array}$ & 73 & 78 & 74 \\
$\begin{array}{l}\text { Movimentos de } \\
\text { estoque }\end{array}$ & 110 & 94 & 181 \\
$\begin{array}{l}\text { Movimentos } \\
\text { debacklog }\end{array}$ & 49 & 33 & 13 \\
$\begin{array}{l}\text { Tempo (s) } \\
\text { Qtd. De }\end{array}$ & $<0,1$ & $<0,1$ & $<0,1$ \\
Veículos & 26 & 26 & 28 \\
\hline
\end{tabular}

na prática de uma transportadora de carga parcelada pelo modal rodoviário no Brasil.

Os experimentos computacionais foram realizados em um laptop com CPU Intel(R) Core (TM) 2 DU0 T6600 2,2GHz, e $4 \mathrm{~GB}$ de memória RAM (somente um processador foi utilizado) e sistema operacional Windows 7 Ultimate. Neste estudo, exemplares dos modelos foram resolvidos utilizando o pacote de otimização CPLEX versão 11.1.1 (llog, 2008). A linguagem de modelagem AMPL (Fourer et al., 2002) foi utilizada para facilitar a interface entre os modelos e o CPLEX. Os resultados obtidos ao se resolver os problemas aleatórios gerados para cada modelo são apresentados na Tabela 3. Nessa tabela pode-se verificar que, conforme o tamanho do problema aumenta, aumentam também os tempos de execução, porém sendo ainda bem aceitáveis para decisões práticas.

Verificaram-se os efeitos na solução final quando da variação de determinados parâmetros, apresentando, para alguns modelos, uma relação direta entre a mudança de determinado parâmetro e seu impacto (positivo ou negativo) na solução. Os modelos se comportaram de forma coerente e consistente com relação a estas mudanças e seus efeitos, tendo:

- Modelo $M_{1}$ : com esse modelo foi possível a obtenção da solução ótima em todos os experimentos computacionais realizados, utilizando dados gerados aleatoriamente, inclusive ao se variar o parâmetro $A_{i j}^{v}$.

- Modelo $M_{2}$ : além da alocação dos veículos vazios, realizou-se também o dimensionamento da frota adicional necessária para que toda a demanda fosse atendida. É importante destacar a importância do parâmetro $C^{v}$, pois as soluções obtidas foram muito sensíveis a esse parâmetro, variando a quantidade adicional de veículos de 7 a 79 veículos. Isso indica que, em aplicações práticas, esse valor deve ser investigado com cautela, sob pena de incorrer em soluções que não condizem com o problema em estudo.

- Modelo $M_{3}$ : foi incluída a restrição de capacidade nos terminais de destino em certos períodos, e o CPLEX se mostrou eficaz para a solução do problema. Foi possível verificar que, conforme já esperado, quanto menor a capacidade nos terminais, maior a quantidade de carga rejeitada, ou não atendida.

- Modelo $M_{4}$ : não há cargas rejeitadas e deseja-se definir a alocação de veículos, considerando-se possíveis cargas em backlog, e que devem ser atendidas até o fim do horizonte de planejamento, tendo uma penalização $\left(h_{i j t}\right)$ para cargas não atendidas no período solicitado. A solução ótima pôde ser obtida em todos os problemas-teste utilizados, podendo evidenciar que quanto maior a penalidade $h_{i j t}$, maior a quantidade de movimentos vazios, e obviamente uma menor quantidade de movimentos de backlog.

- Modelo $M_{5}$ : realiza o dimensionamento da frota adicional necessária para que toda a demanda seja atendida, incluindo restrições de capacidade nos terminais de destino, e permitindo que cargas permaneçam em backlog para atendimento em períodos futuros. Esse modelo pôde ser resolvido utilizando o CPLEX, sendo possível verificar a sensibilidade dos parâmetros $h_{i j t}$ e $C^{v}$ na solução final do problema.

Pôde-se verificar que, de forma geral, quando o problema possui somente dois grupos de veículos ( $|E|=2$ ), i.e., veículos próprios e agregados), o problema pode ser resolvido sem maiores dificuldades utilizando o método branch-and-cut do CPLEX, com um tempo de processamento aceitável na prática. Porém, em situações práticas, pode ser necessário separar os veículos em muitos grupos (no limite, um grupo para cada veículo, para que os veículos sejam tratados individualmente, placa a placa), fazendo com que $|E|$ seja igual à quantidade de veículos disponíveis. Foram gerados aleatoriamente problemas em que cada veículo pertence a um grupo diferente. Para isso, fixou-se $|E|=130$, e cada $m_{i t}^{v}=\{0,1\}, \forall i \in N, \forall t \in T$, $\forall v \in E$, satisfazendo:

$$
\sum_{i \in N} \sum_{t \in T} \sum_{v \in E} m_{i t}^{v}=|E| .
$$

Assim, a complexidade para solução do modelo matemático deve crescer consideravelmente, em função do aumento do número de variáveis e restrições, e ainda devido ao fato de que todas as variáveis de 
Tabela 3. Resultados obtidos com os problemas aleatórios gerados.

\begin{tabular}{|c|c|c|c|c|c|}
\hline Modelo $\boldsymbol{M}_{1}$ & Problema 1 & Problema 2 & Problema 3 & Problema 4 & Problema 5 \\
\hline Função Objetivo & $14.024,58$ & $10.400,93$ & $7.569,51$ & $9.073,29$ & $8.798,15$ \\
\hline Demanda atendida & 213 & 173 & 155 & 166 & 135 \\
\hline Demanda não atendida & 87 & 127 & 145 & 134 & 165 \\
\hline Movimentos cheios & 213 & 173 & 155 & 166 & 135 \\
\hline Movimentos vazios & 374 & 307 & 286 & 310 & 243 \\
\hline Movimentos de estoque & 1224 & 1095 & 1107 & 833 & 948 \\
\hline Tempo (s) & 5,1636 & 2,9172 & 2,9952 & 2,4024 & 2,4648 \\
\hline Qtd. de Veículos & 130 & 130 & 130 & 130 & 130 \\
\hline Modelo $\boldsymbol{M}_{2}$ & Problema 1 & Problema 2 & Problema 3 & Problema 4 & Problema 5 \\
\hline Função Objetivo & 658,31 & 322,75 & 837,11 & 473,41 & 483,02 \\
\hline Demanda atendida & 300 & 300 & 300 & 300 & 300 \\
\hline Demanda não atendida & 0 & 0 & 0 & 0 & 0 \\
\hline Movimentos cheios & 300 & 300 & 300 & 300 & 300 \\
\hline Movimentos vazios & 201 & 99 & 264 & 244 & 223 \\
\hline Movimentos de estoque & 4448 & 5820 & 3092 & 2987 & 4021 \\
\hline Tempo (s) & 3,9624 & 3,4944 & 4,3524 & 3,9780 & 9,1261 \\
\hline Qtd. de Veículos & 235 & 289 & 199 & 198 & 217 \\
\hline Modelo $\boldsymbol{M}_{3}$ & Problema 1 & Problema 2 & Problema 3 & Problema 4 & Problema 5 \\
\hline Função Objetivo & $14.024,58$ & $10.400,93$ & $7.569,51$ & $9.073,29$ & $8.798,15$ \\
\hline Demanda atendida & 213 & 173 & 155 & 166 & 135 \\
\hline Demanda não atendida & 87 & 127 & 145 & 134 & 165 \\
\hline Movimentos cheios & 213 & 173 & 155 & 166 & 135 \\
\hline Movimentos vazios & 374 & 307 & 286 & 310 & 243 \\
\hline Movimentos de estoque & 1224 & 1095 & 1107 & 833 & 948 \\
\hline Tempo (s) & 10,6705 & 6,1152 & 6,1308 & 4,7736 & 5,1324 \\
\hline Qtd. de Veículos & 130 & 130 & 130 & 130 & 130 \\
\hline Modelo $\boldsymbol{M}_{4}$ & Problema 1 & Problema 2 & Problema 3 & Problema 4 & Problema 5 \\
\hline Função Objetivo & $21.227,25$ & $19.579,52$ & $16.573,93$ & $15.758,38$ & $19.785,32$ \\
\hline Demanda atendida & 300 & 300 & 300 & 300 & 300 \\
\hline Demanda não atendida & 0 & 0 & 0 & 0 & 0 \\
\hline Movimentos cheios & 300 & 300 & 300 & 300 & 300 \\
\hline Movimentos vazios & 401 & 498 & 372 & 323 & 363 \\
\hline Movimentos de estoque & 924 & 427 & 453 & 243 & 1 \\
\hline Movimentos de backlog & 2148 & 2630 & 2880 & 2461 & 4511 \\
\hline Tempo (s) & 5,5848 & 3,3540 & 6,1776 & 3,9624 & 2,2464 \\
\hline Qtd. de Veículos & 130 & 130 & 130 & 130 & 130 \\
\hline Modelo $\boldsymbol{M}_{5}$ & Problema 1 & Problema 2 & Problema 3 & Problema 4 & Problema 5 \\
\hline Função Objetivo & 619,85 & 315,55 & 780,34 & 447,48 & 448,41 \\
\hline Demanda atendida & 300 & 300 & 300 & 300 & 300 \\
\hline Demanda não atendida & 0 & 0 & 0 & 0 & 0 \\
\hline Movimentos cheios & 300 & 300 & 300 & 300 & 300 \\
\hline Movimentos vazios & 209 & 101 & 263 & 235 & 203 \\
\hline Movimentos de estoque & 3892 & 5661 & 2766 & 2833 & 3587 \\
\hline Movimentos de backlog & 64 & 22 & 111 & 67 & 78 \\
\hline Tempo (s) & 4,9140 & 4,2276 & 24,7106 & 9,2977 & 9,1573 \\
\hline Qtd. de Veículos & 216 & 282 & 189 & 186 & 201 \\
\hline
\end{tabular}

decisão do problema, por exemplo, para o modelo $M_{1}$, se tornam binárias. 0 problema realista em estudo, contendo 53 terminais, 36 períodos e 130 veículos, resulta em um modelo com mais de 26 milhões de variáveis de decisão binárias e restrições, tornando o problema difícil de ser resolvido na sua otimalidade, mesmo utilizando pacotes comerciais de otimização de última geração. lsso pode inviabilizar a solução do problema pelo CPLEX, em função dos requisitos de tempo e memória computacionais, o que sugere a necessidade de desenvolvimento de métodos heurísticos para solução. 


\section{Conclusões e perspectivas}

Neste artigo foi abordado o PADV no contexto de transporte rodoviário de cargas completas entre terminais. Foram propostos cinco modelos matemáticos para tratar não só o problema de alocação dinâmica de veículos, mas também para incorporar nos modelos decisões de alocação de frota adicional necessária para que toda a demanda seja atendida. Os modelos propostos consideraram ainda a possibilidade de atrasos decorrentes da possível falta de veículos e penalizações por demandas não atendidas no período solicitado. Foram realizados experimentos computacionais utilizando os modelos propostos para analisar seus comportamentos ao resolver um conjunto de problemas gerados aleatoriamente, de tamanho e complexidade similares aos encontrados em uma empresa típica de transporte rodoviário de cargas parceladas no Brasil.

Os resultados teóricos indicam que esses modelos podem ser úteis para apoiar decisões no dia a dia de transportadoras brasileiras de carga parcelada, pois tendo-se os dados de entrada no início do horizonte de planejamento, usualmente no primeiro dia da semana, e ainda o posicionamento dos veículos no momento do planejamento, por exemplo via GPS (Global Positioning System), é possível utilizar o modelo $M_{1}, M_{3}$ ou $M_{4}$ para verificar o atendimento das demandas com a frota disponível e, se houver demandas não atendidas, analisar via modelos $M_{2}$ e $M_{5}$ a possibilidade de contratação de veículos adicionais para atender a todas as demandas, levando-se em conta o trade-off entre o custo de incremento de novos veículos e o custo de backlog de cargas.

Como perspectiva para trabalhos futuros pretende-se desenvolver e aplicar heurísticas e metaheurísticas para a solução do PADV, por exemplo, simulated annealing (Kirkpatrick et al., 1983) e colônia de formigas (Ant Colony Optimization) (Dorigo \& Stützle, 2004), para o caso especial em que a frota necessita ser separada em uma grande quantidade de grupos, ou, no limite, placa a placa. Pretende-se ainda realizar um processo de validação prática dos modelos e dos métodos de solução propostos em ambientes reais de operação em uma empresa de transporte rodoviário de cargas parceladas no Brasil. Outra linha de pesquisa interessante seria estender os modelos e estudar abordagens de solução para considerar parâmetros incertos no problema, particularmente nas demandas.

\section{Agradecimentos}

Os autores agradecem aos revisores anônimos pelos úteis comentários e sugestões de revisão no texto, e à Patrus Transportes Urgentes Ltda. pela importante colaboração e apoio a esta pesquisa. Este estudo também contou com apoio parcial do CNPq e FAPESP.

\section{Referências}

Beaujon, G. J., \& Turnquist, M. A. (1991). A model for fleet sizing and vehicle allocation. Transportation Science, 25(1), 19-45. http://dx.doi.org/10.1287/trsc.25.1.19.

Birge, J. R., \& Louveaux, F. (1997) Introduction to stochastic programming (Springer Series in Operations Research and Financial Engineering, s. 1). Switzerland: Springer.

Bojovic, N. J. (2002). A general system theory approach to rail freight car fleet sizing. European Journal of Operational Research, 136(1), 136-172. http://dx.doi.org/10.1016/ S0377-2217(01)00034-0.

Braklow, J. W., Graham, W. W., Hassler, S. M., Peck, K. E., \& Powell, W. B. (1992). Interactive optimization improves service and performance for yellow freight system. Interfaces, 22(1), 147-172. http://dx.doi.org/10.1287/inte.22.1.147.

Dejax, P. J., \& Crainic, T. G. (1987). A review of empty flows and fleet management models in freight transportation. Transportation Science, 21(4), 227-247. http://dx.doi. org/10.1287/trsc.21.4.227.

Dorigo, M., \& Stützle, T. (2004). Ant Colony Optimization. Cambridge: The MIT Press. http://dx.doi.org/10.1007/b99492.

Fourer, R., Gay, D. M., \& Kernighan, B. W. (2002). AMPL: a modeling language for mathematical programming. Pacific Grove: Duxbury Press.

Frantzeskakis, L. F., \& Powell, W. B. (1990). A successive linear approximation procedure for stochastic, dynamic vehicle allocation problems. Transportation Science, 24(1), 40-57. http://dx.doi.org/10.1287/trsc.24.1.40.

Ghiani, G., Laporte, G., \& Musmanno, R. (2003). Introduction to logistics systems planning and control (Wiley-Interscience series in systems and optimization). Hoboken: John Wiley \& Sons. http://dx.doi.org/10.1002/0470014040.

Hall, R. W. (1999). Stochastic freight flow patterns: implications for fleet optimization. Transportation Research Part A, Policy and Practice, 33(6), 449-465. http://dx.doi.org/10.1016/ S0965-8564(98)00063-9.

Hall, R. W., \& Zhong, H. (2002). Decentralized inventory control policies for equipment management in a manyto-many network. Transportation Research Part A, Policy and Practice, 36(10), 849-865. http://dx.doi.org/10.1016/ S0965-8564(01)00025-8.

llog. (2008). Ilog CPLEX 11.1.1: user's manual. Florianópolis: llog.

Kirkpatrick, S., Gelatt Junior, C. D., \& Vecchi, M. P. (1983). Optimization by simulated annealing. Science, 220(4598), 671-680. http://dx.doi.org/10.1126/science.220.4598.671. PMid:17813860.

Oliveira, P. F., \& Pizzolato, N. D. (2002). A eficiência da distribuição através da prática do crossdocking. In Anais do XXII Encontro Nacional de Engenharia de Produção, Curitiba.

Powell, W. B. (1986). A stochastic model of the dynamic vehicle allocation problem. Transportation Science, 20(1), 117-129. http://dx.doi.org/10.1287/trsc.20.2.117.

Powell, W. B. (1987). An operational planning model for the dynamic vehicle allocation problem with uncertain demands. 
Transportation Research Part B: Methodological, 21(3), 217-232. http://dx.doi.org/10.1016/0191-2615(87)90005-1.

Powell, W. B. (1988). A comparative review of alternative algorithms for the dynamic vehicle allocation problem. In B. L. Golden \& A. Assad (Eds.), Vehicle routing: methods and studies (Studies in management science and systems, Vol. 16, pp. 249-291). Amsterdam: Elsevier Science Publisher.

Powell, W. B. (1996). A stochastic formulation of the dynamic assignment problem, with an application to truckload motor carrier. Transportation Science, 30(3), 195-219. http://dx.doi.org/10.1287/trsc.30.3.195.

Powell, W. B., \& Carvalho, T. A. (1998a). Dynamic control of logistics queuing networks for large-scale fleet management. Transportation Science, 32(2), 90-109. http://dx.doi. org/10.1287/trsc.32.2.90.

Powell, W. B., \& Carvalho, T. A. (1998b). Real-time optimization of containers and flatcars for intermodal operations. Transportation Science, 32(2), 110-126. http://dx.doi. org/10.1287/trsc.32.2.110.

Powell, W. B., Jaillet, P., \& Odoni, A. R. (1995). Stochastic and dynamic networks and routing. In M. 0. Ball, T. L. Magnanti, C. L. Monna \& G. L. Nemhauser (Eds.), Handbooks in operations research and management science (Vol. 8, pp. 141-295). Amsterdam: Elsevier Science.
Powell, W. B., Sheffi, Y., \& Thiriez, S. (1984). The dynamic vehicle allocation problem with uncertain demands. In 9th International Symposium on Transportation and Traffic Theory, Netherlands.

Sayarshad, H. R., \& Ghoseiri, K. (2009). A simulated annealing approach for the multi-periodic rail-car fleet sizing problem. Computers \& Operations Research, 36(6), 1789-1799. http://dx.doi.org/10.1016/j.cor.2008.05.004.

Sayarshad, H. R., \& Tavakkoli-Moghaddam, R. (2010). Solving amulti periodic stochastic model of the rail-car fleet sizing by two-stage optimization formulation. Applied Mathematical Modelling, 34(5), 1164-1174. http://dx.doi. org/10.1016/j.apm.2009.08.004.

Vasco, R. A. (2012). Otimização na alocação dinâmica de veículos no transporterodoviário de cargas completas entre terminais (Tese de doutorado). Departamento de Engenharia de Produção, Universidade Federal de São Carlos, São Carlos.

Vasco, R. A., \& Morabito, R. (2014). Otimização na alocação dinâmica de veículos no transporte rodoviário de cargas completas entre terminais. Gestão \& Produção, 21(2), 271284. http://dx.doi.org/10.1590/S0104-530X2014005000011.

\title{
Sizing and dynamic allocation of vehicles for road transportation of full truckloads between terminals
}

\begin{abstract}
This paper addresses the dynamic vehicle allocation problem (DVAP) involving the road transportation of full loads between terminals, including the determination of the number of additional vehicles required to meet the demand for transportation in a multi-period finite planning horizon. The DVAP consists of defining the "movements" of a fleet of vehicles that transport goods between terminals with a wide geographical distribution and interact among themselves. These movements may be of fully laden vehicles, unladen vehicles used for repositioning, or vehicles held at a terminal to meet future demands. Emphasis is given to the characterization of the problem in real situations, the mathematical modeling of the problem by means of integer linear programming and the use of operational research techniques in solving the problem. The computational experiments conducted indicate that this approach can be useful for the solution of problems encountered in everyday life of a LTL transportation company in Brazil.
\end{abstract}

\section{Keywords}

Dynamic vehicle allocation. Multi-period integer linear optimization. Freight transportation. Full truck loads. 